\section{Cahiers de Narratologie}

Analyse et théorie narratives

$35 \mid 2019$

Le style comme événement

\title{
Instabilités narratives
}

John Pier

\section{OpenEdition}

Journals

Electronic version

URL: http://journals.openedition.org/narratologie/9508

DOI: 10.4000/narratologie.9508

ISSN: 1765-307X

\section{Publisher}

LIRCES

\section{Electronic reference}

John Pier, «Instabilités narratives », Cahiers de Narratologie [Online], 35 | 2019, Online since 03

September 2019, connection on 15 November 2019. URL : http://journals.openedition.org/ narratologie/9508; DOI : 10.4000/narratologie.9508

This text was automatically generated on 15 November 2019.

Article L.111-1 du Code de la propriété intellectuelle. 


\title{
Instabilités narratives
}

\author{
John Pier
}

1 Cet article est tiré d'une conférence donnée le 20 novembre 2018 par John Pier dans le séminaire "Recherches contemporaines en narratologie ». Le programme du séminaire du CRAL est accessible sur le site : https://enseignements-2018.ehess.fr/2018/ue/2733/ Pour donner une idée initiale de ce que j'appelle les instabilités narratives, je commencerai par un exemple tiré de Pale Fire de Vladimir Nabokov (1962; traduction française 1965). Ce roman consiste en une "Introduction », un poème inachevé d'une trentaine de pages (il ne manque que le dernier vers) intitulé «Feu pâle » par John Shade, un «Commentaire» de 200 pages sur le poème par un certain James Kinbote et un «Index ». La première strophe se termine par les mots « cette terre de cristal» (v. 12). Il n'y a pas de renvoi de note. Le «Commentaire» propose deux pages d'éclaircissements sur ces mots, éclaircissements qui, on s'en aperçoit très vite, n'ont qu'un rapport éloigné avec le poème commenté. Il commence ainsi :

Vers 12 : cette terre de cristal

Peut-être une allusion à la Zembla, ma chère patrie. Sur le brouillon décousu et à moitié effacé que je ne suis pas du tout certain d'avoir déchiffré correctement suivent les vers :

Ah, je ne dois pas oublier de dire quelque chose

Que mon ami m'a raconté à propos d'un certain roi. (Nabokov 1965 : 66)

2 Alors que John Shade, universitaire américain, produit un poème méditatif et autobiographique sur un ton qui rappelle l'œuvre du poète classique anglais du XVIIIe siècle, Alexander Pope (plus spécifiquement son Essai sur l'homme), Kinbote fait le portrait d'un pays mythologique lointain, Zembla, dont il serait le roi déchu en exil, le Roi Charles. En commentant le poème de Shade, "Feu pâle", Kinbote, tout en prétendant être le Roi Charles, écrit une sorte d'autobiographie de ce dernier. Les deux diégèses, celle du poème et celle $d u$ "Commentaire ", sont dans une grande mesure indépendantes l'une de l'autre, mais non sans points de contact parfois parlants. Le titre du poème, identique à celui du roman, est tiré de Timon d'Athènes de Shakespeare, et on ne saurait guère dire quel texte est le «feu pâle » de l'autre, une incertitude renforcée par le fait que le nom du poète, Shade, signifie «ombre». Une critique a 
décrit le roman de Nabokov comme un "livre de miroirs », et ce n'est pas un hasard si Shade parle de « combinaisons délectables » (« combinational delight ») :

Je crois comprendre

L'existence, ou du moins une très faible part

De ma propre existence uniquement à travers mon art,

En termes de combinaisons délectables... (v. 971-974)

3 Feu pâle, comme l'œuvre de Nabokov généralement, se caractérise par un fascinant tissu combinatoire et intertextuel d'une densité hors norme. L'unique exemple que j'ai donné, avec ses multiples ramifications, mérite à lui seul un commentaire qui pourrait faire l'objet de toute une intervention. D'ailleurs - et c'est ce qui nous intéressera ici la situation n'est guère simplifiée par le fait que le lecteur est ballotté près de 130 fois entre le poème et le « Commentaire ", sans la moindre assistance de renvois de notes au niveau du poème, pour consulter des remarques dont certaines vont jusqu'à une dizaine de pages, portant a priori sur des fragments de quelques mots tirés du poème ${ }^{1}$. Dans Feu pâle, la fameuse linéarité du texte est violemment mise en question.

4 C'est à partir de cet exemple que je souhaite maintenant me pencher sur le problème des instabilités narratives et sur quelques questions annexes, afin de défendre l'idée que le discours narratif est un système complexe ou dynamique. Dans cette perspective, le récit est caractérisé par ce que les spécialistes des sciences de la complexité appellent des structures dissipatives. Que signifie cette expression qu'on pourrait prendre pour un oxymore? Par structures dissipatives, Ilya Prigogine et Isabelle Stengers entendent un processus entropique par lequel un système évolue vers le désordre. Selon eux, cette évolution est le résultat de l'interaction d'un système avec le monde extérieur, son imbrication dans des conditions éloignées de l'équilibre. Pourtant, ce système, une fois éloigné de l'équilibre, produit un ordre nouveau: «la dissipation d'énergie et de matière - généralement associée aux idées de perte de rendement et d'évolution vers le désordre - devient, loin de l'équilibre, source d'ordre ; la dissipation est à l'origine de ce qu'on peut bien appeler de nouveaux états de la matière " (Prigogine et Stengers 1979: 216). Ou encore: les structures dissipatives sont «des structures spatiotemporelles qui surgissent dans des conditions loin de l'équilibre» (Prigogine 1997 : 202). Dans des structures de ce genre, l'instabilité joue un rôle clef. Mais - et c'est important de le souligner - dans le contexte des sciences de la complexité, le principe d'instabilité possède un sens qui ne correspond que de façon imprécise à ce qu'on comprend dans la langue de tous les jours. Pour le spécialiste de la complexité, l'instabilité est le produit de trois facteurs: la non linéarité, le feedback positif et les bifurcations. En plus, ces trois facteurs sont indissociables des conditions de quasi équilibre et de loin de l'équilibre étudiées par la thermodynamique. Mon argument est que ces différents concepts, dont les origines se trouvent dans une suite d'évolutions des sciences naturelles depuis le début du XIXe siècle (et qui forment elles-mêmes un chapitre fort intéressant dans l'histoire culturelle) ont un certain nombre de corrélats qui sont potentiellement productifs pour l'élaboration de la théorie narrative, et qui méritent donc d'être approfondis² .

5 Mais avant de traiter de ces questions de manière plus détaillée, il convient de mettre en perspective la confrontation entre deux domaines aussi éloignés, à première vue, que le récit et les sciences de la complexité. Prenons, par exemple, les différentes propositions de créer une "science de la littérature». Ces initiatives sont souvent dénoncées comme autant de tentatives de déshumaniser la littérature, comme s'il s'agissait de transformer la littérature en une science exacte ou expérimentale. Quels 
que soient les mérites de telles critiques, des recherches transdisciplinaires fructueuses dans de nombreux domaines depuis quelques décennies nous donnent raison de tempérer ce genre de jugement, comme on peut le constater, dans le domaine qui nous concerne: la narratologie. En effet, un nombre non négligeable de spécialistes de psychologie cognitive, de sociologie, d'intelligence artificielle, des sciences de la communication, de droit, de psychothérapie, de médecine (il existe aujourd'hui une discipline qui s'appelle "médecine narrative", enseignée dans quelques facultés de médecine), pour ne donner que quelques exemples, s'intéressent à différentes questions débattues par les narratologues. Et le titre de ce séminaire - «Hasard, causalité, contingence dans le récit»- témoigne bien de ce décloisonnement des disciplines, car ces catégories ne sont pas la réserve exclusive de quelque discipline que ce soit. En fait, il s'agit de catégories qui suscitent des interrogations qui nous obligent à traverser les frontières et, dans notre cas, à réfléchir sur le rôle culturel, social et cognitif du récit ainsi que sur le statut disciplinaire de la théorie narrative.

6 Pour ma part, quand j'ai commencé à m'intéresser aux sciences de la complexité, je me sentais un peu comme Monsieur Jourdain: en faisant des travaux de narratologie, je travaillais de façon indirecte, sans le savoir, avec un certain nombre de concepts et de principes courants parmi les spécialistes de ces sciences. Je pense notamment aux problématiques de la narrativité, des configurations narratives, des niveaux narratifs, des mondes narratifs, de l'intertextualité, de l'intermédialité et de la séquentialité. Mais ces questions-là resteront en marge de mes propos d'aujourd'hui - sauf la séquentialité, sur laquelle je reviendrai.

7 Précisons tout d'abord que la narratologie ne figure pas parmi les sciences de la complexité. En fait, il n'existe pas de discipline bien définie qui s'appelle théorie de la complexité. Il s'agit plutôt d'une fédération ad hoc de disciplines qui comprennent certains aspects des sciences exactes, des sciences de la vie et des sciences sociales, mais aussi de la météorologie, de l'immunologie, de la démographie, de la bourse des valeurs, etc. Cependant, ces domaines partagent entre eux un certain nombre de principes et de caractéristiques qui se manifestent de différentes manières selon le domaine ou le phénomène en question. Parmi ces caractéristiques communes, qui sont interdépendantes entre elles, se trouvent l'irréductibilité d'un ensemble à des unités de plus en plus petites, l'irréversibilité des processus, la non linéarité et l'imprévisibilité. En confrontant la narratologie et la théorie de la complexité - théorie qui est ellemême issue de la recherche transdisciplinaire - il ne faut pas céder à la tentation de transposer ces catégories telles quelles aux problèmes du récit. Je pense que le mot de Gregory Currie (dans son livre Narratives and Narrators, 2010) doit être pris en considération ici. Selon lui, si on appliquait à l'analyse du récit les lois de la causalité de façon stricte, il n'y aurait plus de récit. La même remarque vaut pour les principes de la complexité vis-à-vis du récit. Le récit est un système complexe ou dynamique, mais c'est un système qui possède ses propres particularités et qui ne peut être conçu dans les mêmes termes que des phénomènes complexes dans les sciences de la nature ou dans les sciences cognitives.

8 Il me semble donc que le plus raisonnable est de postuler une relation de commensurabilité relative entre la narratologie et la théorie de la complexité. Que signifie le mot commensurabilité dans ce contexte? Prenons un exemple. On dit que la mécanique classique newtonienne est commensurable avec la thermodynamique hors équilibre. Cela veut dire que la thermodynamique hors équilibre répond à des questions 
que son prédécesseur ne peut résoudre, mais non que ce prédécesseur soit invalide là où les solutions qu'il propose restent compatibles avec les critères de la thermodynamique hors équilibre. Une relation analogue peut être constatée pour la théorie littéraire où, par exemple, le modèle organique de la poétique morphologique est partiellement commensurable avec les approches sémiotiques. Un exemple frappant est l'étude de Vladimir Propp, Morphologie du conte (1928), qui représente une synthèse des deux systèmes. On trouve des exemples également dans la recherche en narratologie. Certains considèrent que la narratologie des mondes possibles pratiquée par Thomas Pavel, Marie-Laure Ryan, Lubomír Doležel et d'autres est à quelques égards commensurable avec la narratologie cognitive de Monika Fludernik ou de David Herman. J'ajouterai que certains éléments complexes sont depuis longtemps inhérents à la théorie littéraire et à la théorie narrative. Je pense en particulier aux formalistes russes (notamment aux contributions de Boris Tynianov «Le fait littéraire » [1923] et "De l'évolution littéraire " [1925-1927]³), mais aussi aux écrits de Jouri Lotman de l'école de Tartu dont le dernier livre, L'explosion et la culture (2004), s'inspire en partie des travaux d'Ilya Prigogine. On peut également se référer au Cercle linguistique de Prague, dont les membres ont été les premiers, dès la fin des années 1920, à formuler les principes de la structure. Une des préoccupations de cette école, définitoire du structuralisme pour les Pragois, était la notion de mérologie, ou l'étude abstraite des relations des parties au tout - une question qui coïncide avec quelques-uns des aspects de la théorie de la complexité. Enfin, et sur un autre registre, mais non sans rapport à ces problématiques, se trouvent les relations des principes de la complexité avec des objets aussi divers que la musique et la sculpture. J'ai eu récemment l'occasion de voir les vidéos de quelques entretiens avec Pierre Boulez, où le compositeur décrit ses compositions en employant le terme "explosion" dans un sens proche de celui de structures dissipatives. À une autre occasion, un ami sculpteur m'a expliqué un jour que ses œuvres, faites de bronze pour la plupart, sont à considérer comme des structures dissipatives.

9 Ces différentes perspectives sur les relations entre la narratologie et la théorie de la complexité méritent sans doute une discussion plus circonstanciée 4 . Ici, je les évoque essentiellement pour donner une indication sommaire du cadre conceptuel de mes propos. Revenons donc aux instabilités narratives.

10 Une des caractéristiques fondamentales du récit, quel que soit son support - oral, écrit, gestuel, visuel, numérique - est la séquentialité: séquentialité du raconté, séquentialité du racontant. Les interactions entre ces deux séquentialités ont été théorisées de plusieurs façons : par Gérard Genette, qui examine le temps du récit selon les différents procédés liés à l'ordre, à la durée et à la fréquence ; par Meir Sternberg, qui s'intéresse aux relations interséquentielles qui produisent les effets de suspense, de curiosité et de surprise ; par Paul Ricœur, qui explore le temps vécu - pour ne donner que quelques exemples. Je n'ai pas de commentaires à faire sur ces schémas, qui ont tous fait preuve de rigueur et de pertinence analytique. Ce que je souhaite faire ici, c'est lier le récit au phénomène d'irréversibilité, connu également sous l'expression "la flèche du temps ", tel que ce phénomène a été formulé par la thermodynamique hors équilibre. Dans cette discipline, l'irréversibilité (qu'on trouve par exemple dans les réactions chimiques) joue un rôle important dans la mesure où elle se distingue d'un des principes de base de la mécanique newtonienne : le principe de réversibilité. La réversibilité se réalise à l'image de la pendule qui ne subit pas les effets de la friction et qui, a priori, bascule dans un sens et dans l'autre de façon perpétuelle et déterministe et donc calculable. Les 
processus irréversibles, par contre, fonctionnent de façon stochastique ou aléatoire, et ils sont donc imprévisibles, comme la trajectoire d'un ballon qui se dégonfle. Selon Nicolis et Prigogine (1992: passim), la redéfinition des relations entre le réversible et l'irréversible et entre le déterministe et le stochastique représente un développement majeur des sciences du XXe siècle.

11 Ces considérations ne manquent pas de pertinence pour le récit. Dans Feu pâle, comme dans tout discours narratif, le processus de réception est irréversible. Tant que la lecture de ce livre avance de façon linéaire, de la première à la dernière page, ce processus reste plus ou moins déterministe. La grammaticalité des phrases du poème, sa prosodie avec sa présentation sous la forme de strophes, sa division en quatre « chants » évoquant les « Four Quartets » de T.S. Eliot, etc. - tous ces éléments reposent sur des régularités formelles et conventionnelles reconnues et sont donc, dans une certaine mesure, prévisibles. Mais tout change à partir du moment où, en tombant sur les mots «cette terre de cristal», non accompagnés d'un renvoi de note, le lecteur se trouve projeté vers un commentaire 35 pages plus loin, qui injecte dans ces mots des développements baroques qui s'écartent des propos du poète, John Shade, dans un récit autodiégétique fait par un individu écrivant sous un pseudonyme. Ici, on est clairement face à un divorce entre le linéaire, dont la logique dit effectivement « un événement $\mathrm{A}$ provoque un événement $B$ ", et le stochastique, qui est non déterministe, probabiliste, voire aléatoire. À un niveau, le poème possède un début, un milieu et une fin, certes, mais à un autre niveau, sous l'effet des multiples irruptions dans le déroulement du poème par les notes en fin de texte - l'apparatus criticus, selon Kinbote, le commentateur - le poème éclate en morceaux, déplacé par une diégèse qui est à la dérive par rapport à celle du poème. Mais tout ne s'arrête pas là, car dans le processus irréversible de la lecture, on revient à la fin de chaque note en fin de texte au poème. Par conséquent, le réversible n'est pas purement et simplement annulé par l'irréversible, mais continue d'exercer une influence structurante sur le roman dans son ensemble. Au fond, l'organisation séquentielle de Feu pâle met en relief une série d'opérations qui sont latentes ou implicites dans tout récit.

12 Cela nous amène maintenant à une autre question, celle de l'équilibre. En narratologie, la séquence est souvent définie en termes d'équilibre et de déséquilibre. C'est le cas de la définition de la séquence narrative proposée par Tzvetan Todorov, définition qui reste généralement reconnue, avec plusieurs variantes, aujourd'hui. Selon Todorov, la séquence

commence par une situation stable qu'une force quelconque vient perturber. Il en résulte un état de déséquilibre; par l'action d'une force dirigée en sens inverse, l'équilibre est rétabli ; le second équilibre est bien semblable au premier, mais les deux ne sont jamais identiques. Il y a par conséquent deux types d'épisodes dans un récit: ceux qui décrivent un état (d'équilibre ou de déséquilibre) et ceux qui décrivent le passage d'un état à l'autre. (Todorov $1973: 82$ )

Cette définition, qui vaut également pour ce que Todorov appelle le "récit idéal », est marquée par les mêmes principes de stabilité, de déterminisme, de réversibilité et de causalité linéaire qui caractérisent la mécanique classique. Elle reflète d'ailleurs le principe de la conservation de l'énergie: selon la première loi de la thermodynamique, dans un système clos, l'énergie cinétique et l'énergie potentielle sont converties l'une dans l'autre de manière continue de sorte que l'énergie totale reste constante et ne diminue pas. De la même manière, la définition todorovienne de la séquence narrative 
postule un mouvement réversible et symétrique entre l'équilibre et le déséquilibre et repose sur le principe du système clos. Suivant cette conception, la séquence (et par extension le récit idéal) est une structure conservatrice plutôt qu'une structure dissipative ou dynamique. Inutile d'insister sur le fait qu'un tel schéma réducteur, appliqué à une œuvre comme Feu pâle, trouve vite ses limites.

15 En regardant maintenant du côté de la thermodynamique hors équilibre, on voit que l'équilibre possède plusieurs nuances qui sont susceptibles d'intéresser le théoricien du récit. Un objet comme le cristal, qui est inerte, atemporel et sans interaction avec l'environnement, est dit être une "structure en équilibre ». Pour l'organisme vivant et pour l'organisation sociale, mais aussi pour le discours narratif - tous deux des systèmes ouverts - un tel équilibre est synonyme de mort. En réalité, les systèmes complexes, y compris le récit, oscillent entre les conditions en "quasi équilibre » en présence de fluctuations minimes et les conditions "loin d'équilibre", où les fluctuations sont amplifiées de manière non linéaire et parfois exponentielle. Le trait distinctif des conditions en quasi équilibre est leur sensibilité aux conditions initiales. Exemple : l'effet papillon, où les battements des ailes d'un papillon à Rio de Janeiro provoquent un ouragan à New York. Dans le cas des récits, les incipits fournissent une large gamme de conditions initiales possibles. Pour en donner un exemple frappant, L'Innommable de Samuel Beckett, afin de souligner la précarité du fait même de raconter, impose une situation loin d'équilibre dès le début : " Où maintenant? Quand maintenant? Qui maintenant? Sans me le demander. Dire je. Sans le penser. Appeler ça des questions, des hypothèses. Aller de l'avant, appeler ça aller, appeler ça de l'avant. » (Beckett 1953 : 7). Les effets de ces lignes se répercutent à travers le roman, du début jusqu'à la fin. En parlant de la séquentialité narrative, il semble donc préférable d'adopter l'idée des oscillations entre des conditions en quasi équilibre et des conditions loin d'équilibre plutôt que du schéma symétrique équilibre - déséquilibre équilibre.

16 Les systèmes qui basculent entre ces deux types de situation sont non seulement des systèmes ouverts, ils sont aussi des systèmes instables. En théorie de la complexité, l'instabilité, comme je l'ai déjà indiqué, est le produit de trois facteurs : la non linéarité, le feedback positif et les bifurcations.

17 La linéarité d'un système est caractérisée par la proportionnalité entre cause et effet: un événement A provoque un événement B. C'est le raisonnement employé par la mécanique classique pour calculer la trajectoire de l'objet individuel - celui d'une planète ou d'une boule de billard, par exemple. On trouve le même type de logique linéaire dans les trente et une fonctions identifiées par Propp dans son analyse du conte ("le héros quitte la maison », "le héros revient », "le héros est récompensé », etc.), dans les «mythèmes » de Lévi-Strauss (« Edipe tue son père », « đEdipe épouse sa mère ", etc.) et dans les grammaires du récit avec ses propositions d'état ("être malade ») et propositions de faire ("guérir»). Du côté des systèmes complexes ou dynamiques, par contre, les causes sont multiples et provoquent des relations asymétriques ou non linéaires, donc disproportionnées, entre les conditions initiales et les résultats. Un domaine qui est particulièrement concerné par ce genre de phénomènes est la météorologie, une discipline qui étudie les comportements à l'échelle large ou planétaire comme le réchauffement climatique, d'une part, et d'autre part les comportements à l'échelle fine comme les orages, les tornades, etc. Les interactions entre ces deux échelles, qui impliquent un vaste nombre de causes et 
d'effets, sont asymétriques et disproportionnées, ce qui rend les prévisions météo généralement délicates.

18 Les problèmes du feedback sont étroitement liés aux problèmes de non linéarité. Historiquement, la cybernétique a été la première discipline à distinguer entre le feedback négatif et le feedback positif. Sous sa forme négative, le feedback vise la stabilité dans les systèmes clos, comme par exemple des dispositifs cybernétiques destinés à réguler les flux de production dans les usines. Dans les systèmes ouverts, par contre, qui se caractérisent par la non linéarité, le feedback est positif : il ne limite pas les fluctuations mais amplifie la disproportionnalité entre les conditions initiales et les résultats, poussant le système loin de l'équilibre vers l'auto organisation et l'émergence de nouveaux systèmes qui, à leur tour, génèrent d'autres cycles de feedback positif. Le feedback positif est bien connu dans la recherche en biologie moléculaire: les organismes vivants produisent de multiples transformations chimiques, mais l'organisation de ces transformations dans le temps et l'espace n'est pas uniforme, ouvrant la voie vers d'autres modes d'organisation.

19 Ces principes du feedback font partie également de la théorie de l'information, et pour cette raison ils concernent le discours narratif. Le feedback négatif se manifeste sous la forme de la redondance, le but étant d'assurer la cohésion microstructurale entre les phrases grâce à l'emploi des noms, des pronoms, des articles, des connecteurs, etc., d'une part, et d'autre part à la cohérence macrostructurale au niveau de l'identité des actants, des actions et des structures sémantiques. Pourtant, l'économie de la communication fait qu'un degré trop élevé de redondance nuira à l'informativité du message et peut, à la limite, la réduire à zéro. L'informativité du message dépend de certains écarts et irrégularités entre ses constituants. Sous l'influence du feedback positif, ces différences sont susceptibles d'être amplifiées, créant des disproportions entre les conditions initiales du message et les effets produits. Dans certains cas, ce processus peut pousser le système loin d'équilibre, menaçant l'intégrité de la communication, mais il peut également déclencher l'émergence de nouveaux messages ou sens.

20 Ce dernier cas de figure est bien illustré par le roman Feu pâle. Les renvois entre le poème et le «Commentaire » créent un système de feedback positif qui fait émerger des sens et des significations qui sont imperceptibles dans le poème. Par exemple, le mot « graduel » vers le début donne lieu dans le «Commentaire » à des remarques sur les noms propres qui ressemblent à ce mot - Jakob Gradus, Jack Degree, Jacques de Gray, James de Gray - les différents alias de l'individu qui, plus tard, sera l'assassin du poète John Shade. C'est comme si ces remarques venaient parasiter le mot "graduel » du poème. Une phrase tirée au livre de Nicolis et Prigogine intitulé À la rencontre $d u$ complexe me semble appropriée pour expliquer ce qui se passe ici : «dans un système non linéaire, le fait d'ajouter une petite cause à une cause qui est déjà présente peut induire des effets dramatiques qui n'ont aucune mesure commune avec l'amplitude de la cause » (Nicolis et Prigogine 1992 : 59).

21 Les instabilités provoquées dans les systèmes complexes par l'intervention des non linéarités et $\mathrm{du}$ feedback positif nous conduisent à une question : quels sont les limites de ce processus ? À partir de quel moment le système se trouve-t-il « au-delà du seuil de stabilité » et "au bord du chaos"? C'est ici qu'intervient le troisième facteur d'instabilité : la bifurcation - un choix entre l'ordre et le désordre. Comme le système est non linéaire et son devenir imprévisible, ce choix ne peut être déterminé ni à partir 
de l'échelle microscopique ni à partir de l'échelle macroscopique. Face à cette situation, les bifurcations produisent de nouvelles formes d'auto-organisation interne et deviennent source d'innovation et de diversification.

Il faut souligner que les bifurcations ne correspondent pas aux structures binaires $\mathrm{du}$ type «Ivan attaque le dragon : il tue le dragon/il ne tue pas le dragon ». Plutôt que des choix ou des alternatifs binaires, les bifurcations sont des briseurs de symétries, liées de façon intrinsèque à la charnière entre les parties du système et l'environnement du système ; elles sont déclenchées aux moments de rencontre de l'« au-delà du seuil de stabilité » et l'« au bord du chaos ». Les bifurcations n'existent pas isolément mais par successions, de manière séquentielle. Entre les bifurcations, les processus avancent de manière déterministe, tandis que la bifurcation, un moment stochastique, un moment de risque, est plus riche en information que les développements entre les bifurcations.

23 La théorie narrative, en toute indépendance des sciences de la complexité, a identifié deux dimensions du récit qui reflètent ces principes. Boris Tomachevski a distingué plusieurs sortes de motifs: les motifs associés, qui forment des liens de succession chronologique et causale entre les événements au niveau de la fable du récit et qui sont habituellement dynamiques; et les motifs libres, des détails et des descriptions qui forment le sujet du récit et qui sont habituellement statiques (Tomachevski 1965 : 272-73). Quarante ans plus tard, Roland Barthes a proposé des distinctions analogues: les fonctions cardinales (ou noyaux), qui constituent l'armature du récit; et les catalyses, qui remplissent l'espace narratif entre les fonctions cardinales (1966). Faute d'avoir suffisamment examiné les questions, je n'insisterai pas trop sur les éventuels parallèles entre les distinctions avancées par Tomachevski et Barthes et la succession de bifurcations et de développements intermédiaires théorisée par les spécialistes de la complexité. Mais cette possibilité, comme tant d'autres, témoigne des relations de commensurabilité entre les théories de la complexité et la narratologie et des apports potentiels de ces relations à la recherche transdisciplinaire dans ce domaine.

24 Ce qui m'intéresse plus ici que les motifs, les fonctions cardinales et les catalyses, c'est comment les bifurcations, briseurs de symétries qui déstabilisent les systèmes, y compris le récit, provoquent des structures dissipatives. Selon Prigogine et Stengers, "L'interaction d'un système avec le monde extérieur, son imbrication dans des conditions de non équilibre, peuvent [...] devenir le point de départ pour la formation de nouveaux états dynamiques de matière - des structures dissipatives " (1984:143). Ou pour le dire autrement: les structures dissipatives émergent de façon imprévue dans des conditions loin d'équilibre, passant du désordre vers un nouvel ordre qui n'est pas inscrit dans le système. Le résultat est un mode d'auto organisation du système caractérisé par l'autonomie, l'adaptabilité aux perturbations et des processus dynamiques loin d'équilibre.

25 Comme illustration narrative de ces principes, on ne pourrait guère mieux tomber que sur Feu pâle de Nabokov. J'ai proposé au début de mon intervention de décrire le récit comme un système complexe formé par des structures dissipatives. La formule convient parfaitement à cette œuvre, caractérisée par ses « combinaisons délectables » ( "combinational delight»). Le titre du roman est le même que celui du poème (en principe, le livre est une édition critique du poème), mais le poème est très largement débordé par le "Commentaire» (ou métatexte) qui est censé porter quelques éclaircissements au poème. En effet, on y lit : « Je n'ai nullement le désir de tordre et de bossuer un apparatus criticus sans ambiguïté en un monstrueux simulacre de roman » 
(Nabokov 1965: 76). Le poème de John Shade devient autant un métatexte du "Commentaire " que le "Commentaire», présenté sous la forme de notes en fin de texte (un paratexte), est un métatexte du poème. L'auteur du "Commentaire", Kinbote, se donne un pseudonyme pour écrire une sorte d'autobiographie de quelqu'un qu'il n'est pas, le dernier roi de Zembla. On finit par se demander: quel texte, finalement, est l'ombre de l'autre? En consultant "L'introduction", on lit qu'il est conseillé au lecteur de lire le « Commentaire » du début à la fin avant de lire le poème. Quant à l'" Index ", ses entrées renvoient très majoritairement aux personnes et aux lieux géographiques mentionnés dans le «Commentaire " plutôt qu'au poème ; mais comme les noms dans l'« Index ", suivis de quelques informations sur les personnes et les lieux concernés, sont présentés en ordre alphabétique, il représente une reconfiguration radicale de la diégèse du "Commentaire ", une histoire en soi plutôt qu'un guide du poème.

Mon propre commentaire sur Feu pâle est qu'il n'est que la représentation pâle d'un commentaire qui pourrait sans doute s'étendre sur un grand nombre de pages dans une tentative vaine de maîtriser les innombrables instabilités narratives dispersées à travers le texte. Mais c'est finalement un roman qui, sous la force de la non linéarité, des nombreuses instances de feedback positif et des bifurcations, éclate dans une auto organisation faite de multiples configurations. Ce qui prédomine dans Feu pâle, ce n'est pas la séquence narrative, définie comme le passage d'un équilibre au déséquilibre suivi d'un retour à l'équilibre, mais bien la séquentialité. La séquence narrative est un dispositif qui couvre certaines configurations locales, mais non le récit dans sa globalité. Il n'y a pas de rapport isomorphe entre la séquence narrative et un supposé "récit idéal ». Quant à la séquentialité, elle fonctionne de manière irréversible, à l'image de la flèche du temps, et elle déclenche des processus non linéaires et stochastiques qui tendent vers des conditions de non équilibre, vers des bifurcations et vers l'auto organisation. Feu pâle est une instance exemplaire mais non isolé de ce phénomène. Ce qui ressort dans cette œuvre, plus qu'ailleurs, ce sont les relations disproportionnées et dissymétriques entre les causes et les effets, entre les conditions initiales et les résultats, entre les parties et le tout.

\section{BIBLIOGRAPHY}

Barthes, Roland (1966), «Introduction à l'analyse structurale des récits », Communications 8 : 1-27. Beckett, Samuel (1953), L'Innommable, Paris, Minuit.

Currie, Gregory (2010), Narratives and Narrators : A Philosophy of Stories, Oxford, Oxford University Press.

Grishakova, Marina, et Maria Poulaki, éd. (2019), Narrating Complexity : Cognition, Embodiment, Evolution, Lincoln, University of NebraskaPress.

Lotman, Jouri Mikhailovotch (2004 [1992]), L'explosion et la culture, Traduit du russe par Inna Merkoulova, Limoges, Presses universitaires de Limoges. 
Nabokov, Vladimir (1965 [1962]), Feu pâle, Traduit de l'anglais par Raymond Girard et MauriceEdgar Coindreau, Paris, Gallimard.

Nicolis, Grégoire, et Ilya Prigogine (1992 [1989]), À la rencontre du complexe, Jacques Chanu et alii (trad.), Paris, Presses universitaires de France.

Pier, John (1992), « Between Text and Intertext : Vladimir Nabokov's Pale Fire », Style 26 (1) : 12-32.

Pier, John (2017), « Complexity : A Paradigm for Narrative? », in Per Krogh Hansen, John Pier, Philippe Roussin et Wolf Schmid (dir.), Emerging Vectors of Narratology, Berlin/Boston, De Gruyter, p. 533-565.

Prigogine, Ilya (1997 [1996], The End of Certainty : Time, Chaos, and the New Laws of Nature, New York, etc., The Free Press.

Prigogine, Ilya, et Isabelle Stengers (1984), Order out of Chaos : Man's new dialogue with nature, Avant-propos d'Alvin Toffler, Londres, Flamingo.

Prigogine, Ilya, et Isabelle Stengers (1979), La nouvelle alliance. Métamorphose de la science, Paris, Gallimard.

Propp, Vladimir (1965 [1928]), Morphologie du conte, suivi de « Les transformations des contes merveilleux » et de E. Mélétinski, «L'étude structurale et typologique du conte », traduit du russe par M. Derrida, T. Todorov et Cl. Kahn, Paris, Seuil.

Todorov, Tzvetan (1982), Poétique, vol. 2 de Qu'est-ce que le structuralisme ? François Wahl (éd.), Paris, Seuil.

Tomachevski, Boris (1965), « Thématique » (1925), in Théorie de la littérature, Textes des Formalistes russes réunis, présentés et traduits par Tzvetan Todorov. Préface de Roman Jakobson, Paris, Seuil.

Tynianov, Iouri (1991), Formalisme et histoire littéraire, Traduit du russe, annoté et présenté par Catherine Depretto-Genty, Lausanne, L'Âge d'Homme.

Walsh, Richard, et Susan Stepney, éd. (2018), Narrating Complexity, Cham, Springer.

\section{NOTES}

1. Pour une analyse de Feu pâle, voir Pier (1992).

2. Pour un exposé plus élaboré, voir Pier (2017).

3. In Tynianov (1991).

4. Deux publications récentes confirment l'intérêt suscité par la perspective complexe sur le récit, notamment Walsh et Stepney (2018) et Grishakova et Poulaki (2019). 